\title{
The CALorimetric Electron Telescope (CALET) on the International Space Station
}

\author{
Yoichi Asaoka* \\ Waseda Research Institute for Science and Engineering, Waseda University, 3-4-1 Okubo, \\ Shinjuku, Tokyo 169-8555, Japan, and \\ JEM Mission Operations and Integration Center, Human Spaceflight Technology Directorate, \\ Japan Aerospace Exploration Agency, 2-1-1 Sengen, Tsukuba, Ibaraki 305-8505, Japan \\ E-mail: yoichi.asaokadaoni.waseda.jp

\section{for the CALET collaboration ${ }^{\dagger}$}

\begin{abstract}
The CALorimetric Electron Telescope (CALET), developed and operated by Japan in collaboration with Italy and the United States, is a high-energy astroparticle physics experiment installed on the International Space Station (ISS). Its mission goals include investigating nearby possible sources of high-energy electrons, elucidating the details of the acceleration and propagation of galactic particles, and detecting potential dark matter signatures. CALET measures cosmic-ray electron+positron flux up to $20 \mathrm{TeV}$, gamma rays up to $10 \mathrm{TeV}$, and nuclei with $\mathrm{Z}=1$ to 40 up to $1,000 \mathrm{TeV}$ for the more abundant elements during long-term observations. CALET has performed continuous observations without major interruptions since mid-October 2015, recording approximately 20 million triggered events per month with energies greater than $10 \mathrm{GeV}$. Here, we present the highlights of CALET observations made over the first three years of operation, namely the electron+positron energy spectrum, the spectra of protons and other nuclei, and gamma-ray observations, including the characterization of on-orbit performance. Some results of the electromagnetic counterpart search for LIGO/Virgo gravitational wave events are also included.
\end{abstract}

36th International Cosmic Ray Conference -ICRC2019-

July 24th - August 1st, 2019

Madison, WI, U.S.A.

\footnotetext{
* Speaker.

${ }^{\dagger}$ for collaboration list see PoS(ICRC2019)1177
} 


\section{Introduction}

The CALorimetric Electron Telescope (CALET) [[] [] ], developed and operated by Japan in collaboration with Italy and the United States, is a highenergy astroparticle physics mission on the International Space Station (ISS).

CALET was launched into orbit aboard the unmanned H2 Transfer Vehicle (HTV) atop the Japanese H2-B carrier rocket on August 19, 2015. At the ISS, CALET was installed on the Japanese Experiment Module-Exposed Facility (JEM-EF). The initial mission was scheduled to last 2 years, with possible extension to 5 or more years. Figure $\square$ shows the JEM with CALET attached at port \#9. CALET has a mostly unobstructed field of view of $45^{\circ}$ from the zenith. Figure Wshows a schematic diagram of the CALET payload.

The main instrument on CALET is a very thick calorimeter that incorporates both imaging and total absorption calorimeters. The overall thickness of CALET for the normal incidence angle is 30 ra-

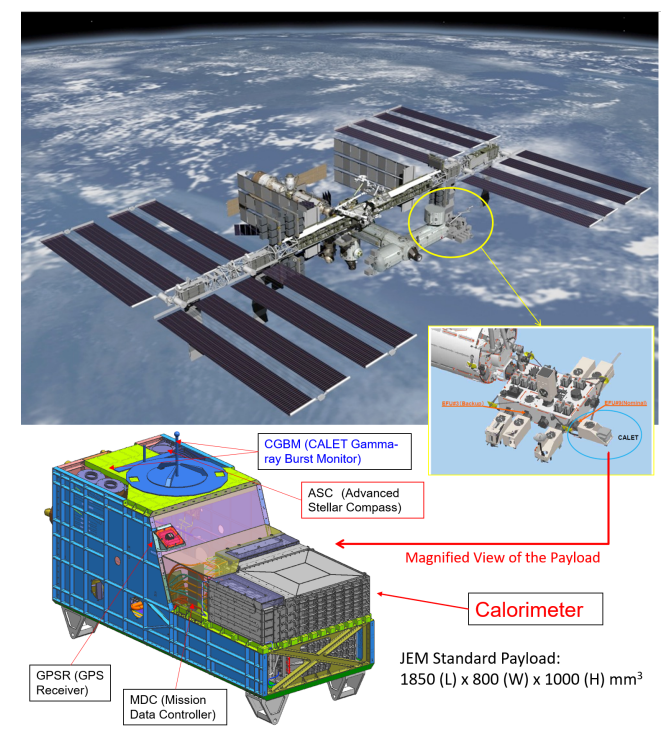

Figure 1: JEM-EF and CALET payload attached at port \#9. The inset shows the CALET instrument package, with the main calorimeter and CALET Gamma-ray Burst Monitor (CGBM) subsystems [四] indicated. diation length, which is equivalent to $\sim 1.3$ proton interaction length. Long-term observations with CALET are conducted using a large-area detector and thus measurements with high statistics are obtained. CALET is designed to find signatures of nearby cosmic-ray accelerators and possibly dark matter in the all-electron (electron+positron) spectrum by high-precision measurement over the broad energy range of $1 \mathrm{GeV}$ to $20 \mathrm{TeV}$, and also for studying the gamma-ray spectrum up to $10 \mathrm{TeV}$. The main components of cosmic rays are protons, helium, and heavier nuclei through iron, which can be measured up to $1 \mathrm{PeV}$. The nuclei spectra measurements are used to investigate the detailed mechanism and parameters that govern the propagation and acceleration of galactic cosmic rays. It is expected that CALET will extend the limits of direct measurements.

\section{Instrument}

The CALET calorimeter (see the right panel of Fig. $\square$ ) consists of a charge detector (CHD), which identifies the charge of the incident particle [四, 回], an imaging calorimeter (IMC), which reconstructs the track of the incident particle and records the initial shower development with fine resolution, and a total absorption calorimeter (TASC), which absorbs the entire energy of the electromagnetic shower particles and identifies the particle species using hodoscopic lead-tungstate crystal arrays. The components and read-out sensors are summarized in the left panels of Fig. $\mathbf{\text { . }}$.

Plastic scintillators arranged in two orthogonal layers, each containing 14 scintillator paddles $\left(3.2 \times 1.0 \times 45.0 \mathrm{~cm}^{3}\right)$, constitute the CHD. These paddles generate photons that are detected by a 
photomultiplier tube (PMT), and the resulting output is sent to a front-end circuit (FEC). This FEC and the readout system that follows it have sufficient dynamic range for detecting particle charges in the range of $Z=1-40$.

The initial shower is resolved by the sampling calorimeter of the IMC, which was carefully designed to accurately determine the shower starting point and incident direction. This calorimeter has a thickness of $3 X_{0}$ and contains five upper $0.2 X_{0}$ and two lower $1.0 X_{0}$ tungsten plates. The IMC contains a total of 16 detection layers, arranged in $8 \mathrm{X}-\mathrm{Y}$ pairs, with each layer segmented into 448 parallel scintillating fibers $\left(0.1 \times 0.1 \times 44.8 \mathrm{~cm}^{3}\right)$, which are individually read out by 64-channel multi-anode PMTs.

The TASC has an overall depth of $27 X_{0}$ and consists of 12 detection layers in an alternating orthogonal arrangement, each comprising 16 lead-tungstate crystal $\left(\mathrm{PbWO}_{4}\right.$ or $\left.\mathrm{PWO}\right)$ logs with dimensions of $2.0 \times 1.9 \times 32.6 \mathrm{~cm}^{3}$. This design allows the TASC to image the development of a shower in three dimensions. With the exception of the first layer, which uses PMTs, a photodiode (PD) in conjunction with an avalanche photodiode (APD) reads the photons generated by each PWO log. Two shaping amplifiers with different gains for each APD (PMT) and PD are used to achieve a dynamic range of $10^{6}\left(10^{4}\right)$.

With these sub-detectors, the trigger system, and the data acquisition system, the CALET instrument has a proton rejection factor of larger than $10^{5}$, a $2 \%$ energy resolution of above $20 \mathrm{GeV}$ for electrons, a very wide dynamic range from $1 \mathrm{GeV}$ to $1 \mathrm{PeV}$, a charge resolution of $0.1-0.3$ electron charge units from protons to above iron (up to $Z=40$ ), an angular resolution of 0.1 to $0.5^{\circ}$, and a geometrical factor on the order of $0.1 \mathrm{~m}^{2} \mathrm{sr}$.

Figure [ [ ] summarizes the CALET particle identification capability. Top-left, top-right, bottomleft, and bottom-right panels show the $3 \mathrm{TeV}$ electron candidate, proton candidate with equivalent shower energy, iron candidate with a shower energy of $9.3 \mathrm{TeV}$, and gamma-ray candidate with $44 \mathrm{GeV}$ of reconstructed energy, respectively. The calorimeter, which has a 30-radiation-length onaxis thickness, absorbs the full electron shower energy, even in the $\mathrm{TeV}$ range. Charge measurement using CHD and IMC separates each of the elements from $Z=1$ to 26 and above. Gamma rays are identified as charge zero because they do not produce a signal before pair creation. Although electrons and protons both have $Z=1$, they can be separated based on differences in their shower shapes. Because of the shower activity in the lower part of TASC due to subsequent interactions of secondary pions, electrons and protons are easily separated by a simple cut even in the $\mathrm{TeV}$
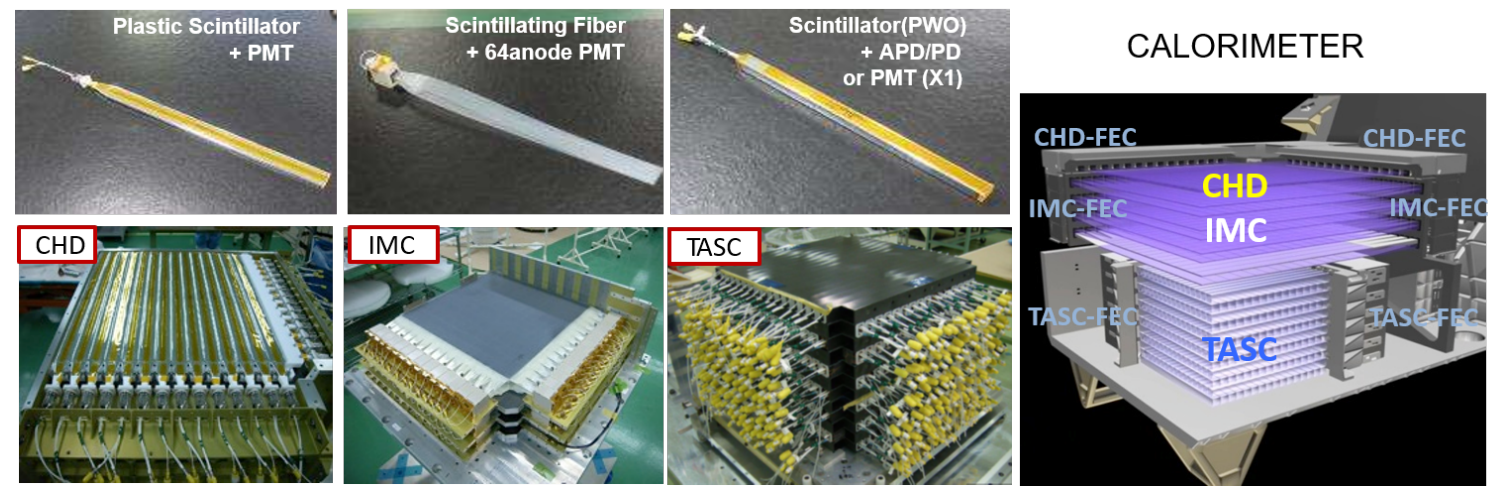

Figure 2: CALET calorimeter, which consists of three subdetectors: a charge detector (CHD), an imaging calorimeter (IMC), and a total absorption calorimeter (TASC). 
region. In addition, various parameters characterizing the shower shape can be utilized to improve separation power [G, 四].
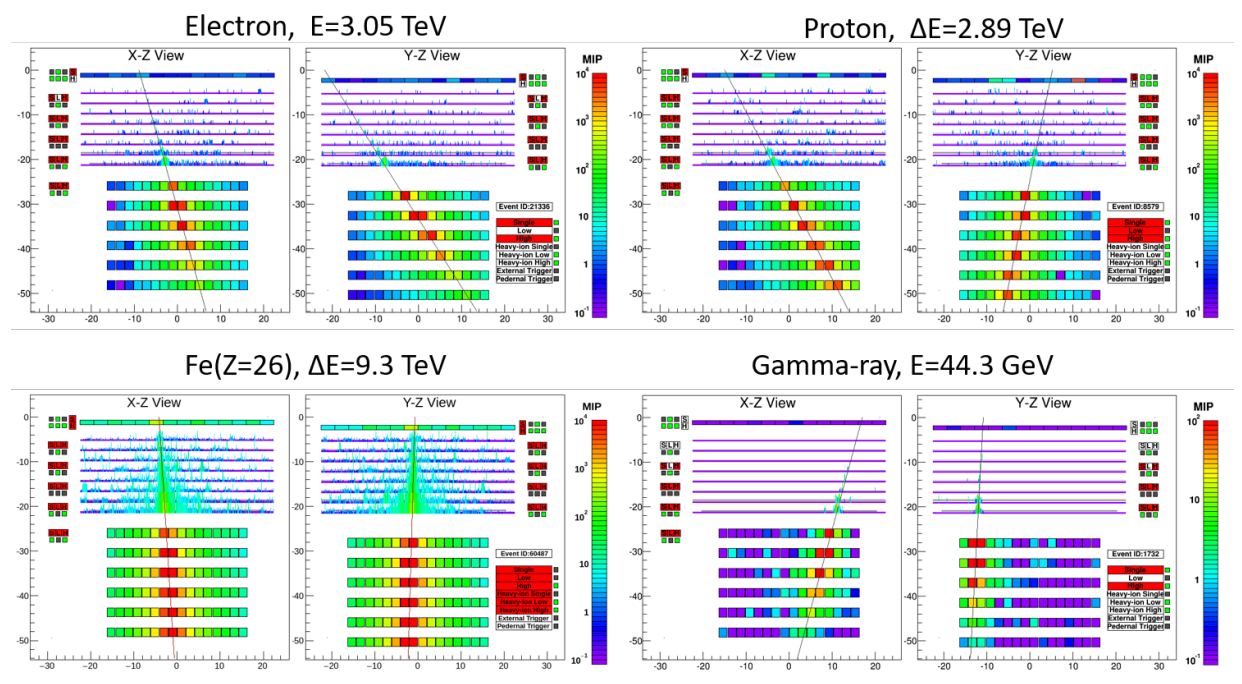

Figure 3: Event examples of high-energy showers collected by CALET onboard the ISS. (Top Left) A $3 \mathrm{TeV}$ electron candidate, (Top Right) a proton candidate with equivalent shower energy, (Bottom Left) an iron candidate with shower energy of $9.3 \mathrm{TeV}$, and (Bottom Right) a $44 \mathrm{GeV}$ gamma-ray candidate.

\section{On-Orbit Operations}

JAXA Ground Support Equipment (JAXAGSE) and the Waseda CALET Operations Center (WCOC) were set up at Tsukuba Space Center and Waseda University, respectively, in order to conduct on-orbit operation of CALET. The transfer of data from the ISS to JAXA utilizes NASA's data relay system. The scientific operations of CALET are planned at WCOC [8] according to parameters as the variation of the geomagnetic rigidity cutoff, which depends on the ISS position. The scheduled command sequences used to control CALET's observation

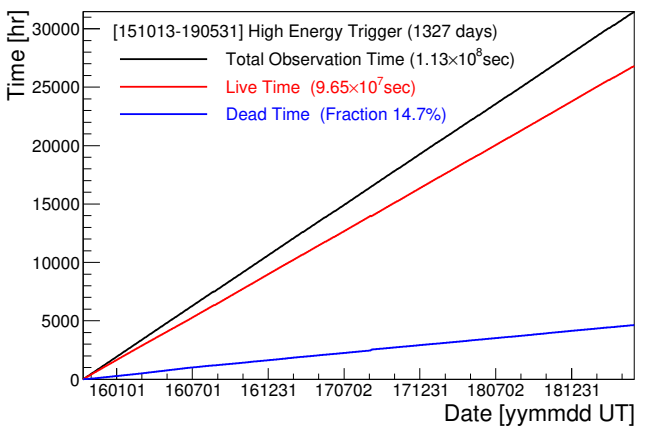

Figure 4: Cumulative observation time (black line) for HE trigger. The red and blue lines indicate the live and dead times, respectively. mode define the time profile of calibration and data acquisition tasks, such as the recording pedestal and penetrating particle events, and the activation and deactivation of observation modes. These modes include a high-geomagnetic-latitude low-energy electron trigger, a low-geomagnetic-latitude low-energy gamma-ray (LE- $\gamma$ ) trigger, and an almost continuously active ultra-heavy trigger mode, during each ISS orbit. A continuously active high-energy (HE; E $>10 \mathrm{GeV}$ ) trigger mode ensures maximum exposure to high-energy electrons and other high-energy shower events.

The total observation time was 1327 days as of May 31, 2019. The live-time fraction was $\sim 84 \%$ for this period. More than 860 million events were observed in HE trigger mode. The accu- 
mulated live time for the HE trigger is plotted in Figure 4 . As shown, the cumulative observation time increased with no significant interruptions since the start of scientific operation in October of 2015. The data transmission from JAXA-GSE to WCOC, as well as the data processing for scientific analysis has also been carried out successfully.

\section{Calibration}

For CALET to achieve highly precise and accurate measurements, energy calibration is required. One important feature of CALET and other thick calorimeter instruments is their excellent energy resolution in the $\mathrm{TeV}$ region. However, calibration errors must still be carefully evaluated and considered when estimating the energy resolution.

Our energy calibration [Q] includes an evaluation of conversion factors between ADC units and energy deposits, confirming linearity over each of the gain ranges (TASC has four gain ranges for each channel), as well as a seamless transition between adjacent gain ranges. Temporal gain variations that occur during long-term observations are also corrected in the calibration [焑].

We have estimated the errors at each calibration step, such as the correction of position and temperature dependence, linear fit procedure
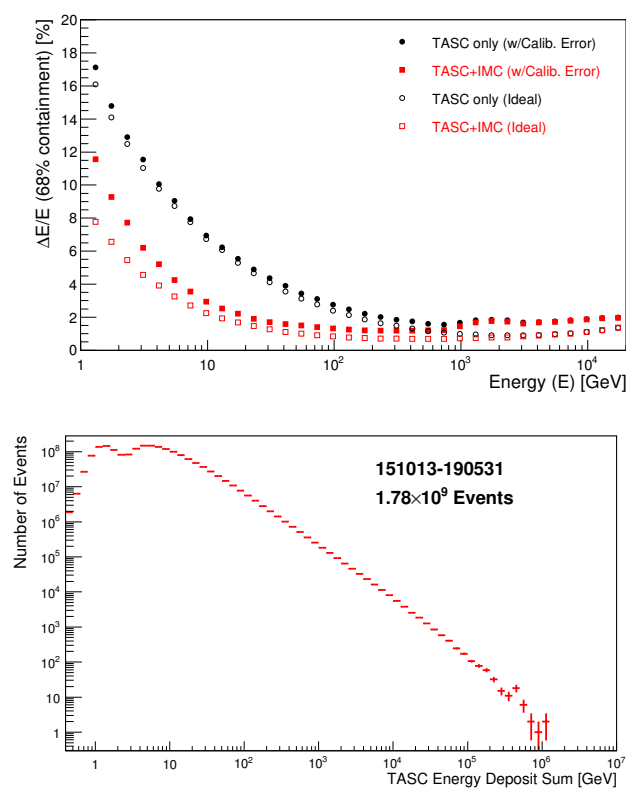

Figure 5: (Top) Energy resolution of electron measurements [Q] and (bottom) TASC energy deposit spectrum based on all triggered events up to May 2019.

of each gain range, gain ratio measurements, and slope extrapolation, as well as the errors inferred from the degree of consistency between energy deposit peaks of non-interacting protons and helium. These errors are included in the estimation of the energy resolution.

As shown in the top panel of Fig. [, this results in a very high resolution of $2 \%$ or better above $20 \mathrm{GeV}$ [Q]. It's worth noting that, even though this calibration is extensive, its uncertainty is a limiting factor for the energy resolution. The intrinsic resolution (i.e., design capability) of CALET is $\sim 1 \%$. In addition, the calibration error in the lower gain ranges are crucial for spectrum measurements in the TeV range.

The TASC energy deposit spectrum based on all triggered events through the end of May 2019 is shown in the bottom panel of Fig. [1. The first and second bumps are due to low- and highenergy triggered events, respectively, whereas the high-energy tail is due to the power-law nature of the cosmic-ray spectrum. This spectrum spans more than six orders of magnitude in energy, from below $1 \mathrm{GeV}$ to above $1 \mathrm{PeV}$. This clearly demonstrates the reliability of CALET energy measurements over a very wide dynamic range. 


\section{Results}

\subsection{Electrons}

\subsubsection{All-Electron Spectrum}

Precise measurement of the all-electron (electron+positron) spectrum in the $\mathrm{TeV}$ region might reveal interesting spectral features that could provide the first experimental evidence of the presence

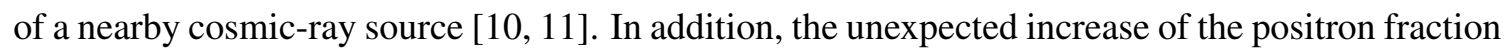
above $10 \mathrm{GeV}$ established by PAMELA [ए2] and AMS-02 [[13] may require a primary source component for positrons in addition to the generally accepted secondary origin. Candidates for such primary sources span from astrophysical (pulsar) to exotic components (dark matter). Since these primary sources emit electron-positron pairs, it is expected that the all-electron spectrum will exhibit a spectral feature near the highest energy range of the primary component.

The CALET collaboration published its first results on electrons in the energy range of $10 \mathrm{GeV}$ to $3 \mathrm{TeV}$ [司]. Subsequently, the DArk Matter Particle Explorer (DAMPE) collaboration published its all-electron spectrum in the energy range of $25 \mathrm{GeV}$ to $4.6 \mathrm{TeV}$ [14]]. Many papers have speculated about the origin of a peak-like structure near $1.4 \mathrm{TeV}$ in the DAMPE data.

An updated version of the CALET all-electron spectrum based on 780 days of flight data and the full geometrical acceptance has recently been published in the energy range of $11 \mathrm{GeV}$ to $4.8 \mathrm{TeV}[\mathrm{⿴囗十}]$. Figure $\mathrm{G}$ shows this updated spectrum using the same energy binning as that used in our previous publication [焑], except for the addition of one extra bin at the high-energy end. The error bars along the horizontal and vertical axes indicate bin width and statistical errors, respectively.

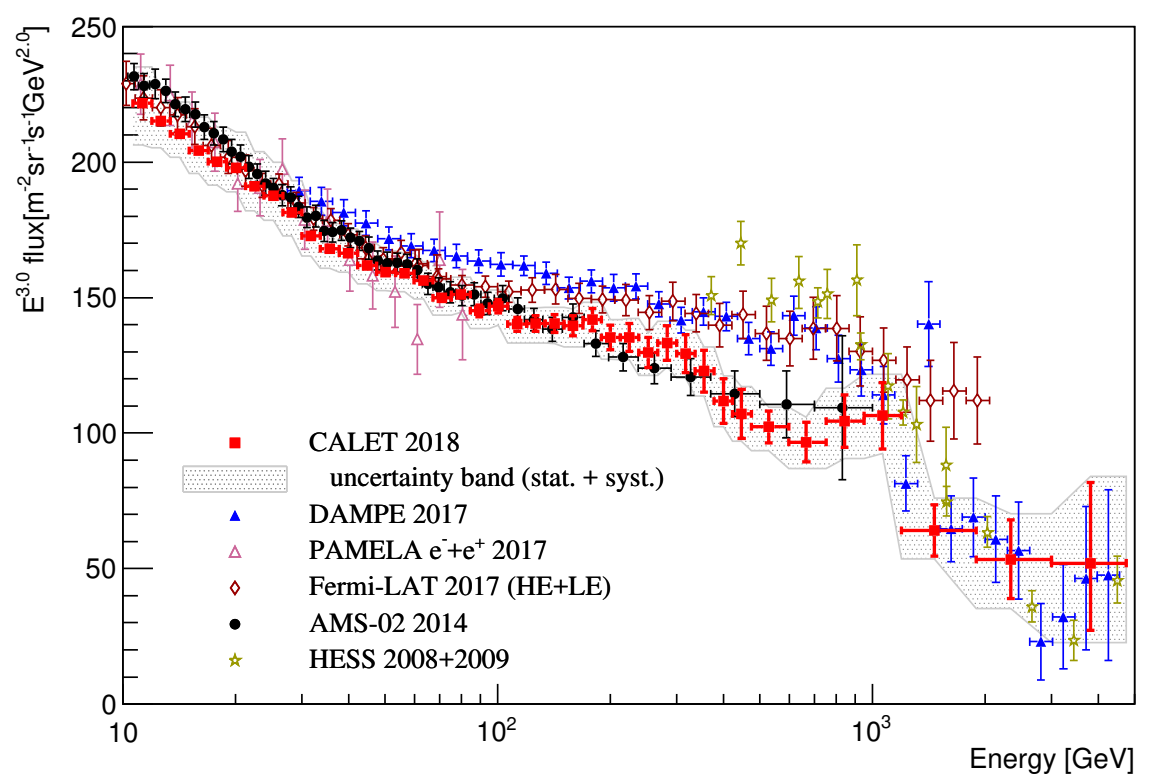

Figure 6: Cosmic-ray all-electron spectrum measured by CALET from $10.6 \mathrm{GeV}$ to $4.75 \mathrm{TeV}$ [四], where the gray band indicates the quadratic sum of statistical and systematic errors. Also plotted are direct measurements in space [ए4, [5], ए6, [1]] and from ground-based experiments [ए8, ए9]. 
The gray band represents the quadratic sum of statistical and systematic errors. Extensive studies on the systematic uncertainties have been performed [ [6,, 0$]$.

Four important implications can be obtained from the current status of the all-electron spectrum measurements. First, CALET's spectrum is consistent with AMS-02 below $1 \mathrm{TeV}$. The two detectors, which can both identify electrons at up to $1 \mathrm{TeV}$, use different detection principles (calorimeter versus magnetic spectrometer), and thus their agreement is an important factual evidence. Second, two groups of measurements, namely AMS-02 + CALET and Fermi-LAT + DAMPE, might suggest the presence of unknown systematic errors, while the situation has been improved since a decade ago. Third, CALET has observed flux suppression that is consistent with DAMPE within errors above $1 \mathrm{TeV}$. Fourth, no peak-like structure was found at $1.4 \mathrm{TeV}$ in CALET data, irrespective of energy binning.

\subsubsection{Anisotropy}

To achieve one of the main goals of CALET, namely to identify the signature of a nearby supernova remnant in electron+positron cosmic rays, the detection of anisotropy could be a smoking-gun signal as well as a spectral feature in the $\mathrm{TeV}$ region. For this purpose, the electron+positron events measured by CALET have been analyzed for possible dipole anisotropy and higher multipole moments. At this conference, methods for deriving the limits of anisotropy from the reconstructed events and the procedures for compensating for the non-uniform sky exposure and inhomogeneous acceptance of the detector will be explained [20]. Furthermore, the preliminary results of the measured anisotropy and upper limits, which depend on threshold energy, will be presented, together with sensitivity estimations and a comparison with the expected anisotropy caused by the Vela supernova remnant [RO]].

\subsection{Hadrons}

Direct measurements of the high-energy spectra of each species of cosmic-ray nuclei up to the PeV energy scale provide information that complements all-electron observations and provide insight into the general conditions of cosmic-ray acceleration and propagation. A possibly chargedependent cutoff in the nuclei spectra is hypothesized to explain the "knee" in the all-particle spectrum. This hypothesis could only be investigated with a space experiment with sufficient exposure. The acceleration limit of supernova remnants calculated with nominal parameters is typically found to be far smaller than the energy of the "knee" [R] observed indirectly by ground detectors. Therefore, precise direct observation of the proton and helium spectra up to $\mathrm{PeV}$ energy is highly important. The spectral hardening observed in the spectra of various nuclei calls for careful investigation. CALET's wide dynamic range ( $\mathrm{GeV}$ to $\mathrm{PeV}$ ) allows the study of features unaffected by systematics from the combination of spectra measured in different experiments. A detailed study will be conducted on the spectral behavior of heavier elements, including secondary-to-primary ratios of up to the $1 \mathrm{TeV} / \mathrm{n}$ energy region, which should yield important information about propagation parameters such as the diffusion coefficient.

\subsubsection{Proton spectrum}

Protons are the most abundant charged particles in cosmic rays. Knowledge of the precise behavior of the proton spectrum is important for understanding the origin, acceleration, and propaga- 
tion of cosmic rays. Therefore, the proton spectrum is investigated in detail based on past observations. The current experimental approaches for the direct measurement of the proton spectrum are based on two main classes of instrument, namely magnetic spectrometers [22, [23] at lower energies, where the presence of a spectral breakpoint has been observed, and calorimeters [24, 207, 26, [27, [28] at higher energies, where the spectrum undergoes hardening. It is of particular interest to determine the onset of spectral hardening and its development in terms of index variation and smoothness parameter (as defined in Ref. [[23]). For a consistent picture, measurements should be unaffected, as much as possible, by systematic errors and a critical comparison of the observations from different experiments is required.

We analyzed flight data collected for 1054 days from October 13, 2015, to August 31, 2018. The total observation live time for high-energy (HE) shower trigger [ $[8]$ is $21421.9 \mathrm{hr}$ and the live-time fraction is $84.7 \%$. In addition, the low-energy (LE) shower trigger operated at a high geomagnetic latitude $[[]]$ is used to extend the energy coverage toward the lower-energy region. Despite a limited live time of $365.4 \mathrm{hr}$, LE data provide sufficient statistics for protons below a few hundred GeV.

To accurately separate protons from helium by charge identification and to minimize helium contamination, it is important to preselect well-reconstructed and well-contained events. Furthermore, by removing events not included in the Monte Carlo (MC) samples, i.e., those with incidence from the zenith angle greater than $90^{\circ}$ and mis-reconstructed events, event samples that are equivalent between flight data and MC data were obtained for charge identification. The event preselection consisted of (1) offline trigger confirmation, (2) geometrical condition, (3) track quality cut, (4) electron rejection cut, (5) off-acceptance event rejection cut, (6) requirement of track consistency with TASC energy deposits, and (7) shower development requirement in IMC. For a detailed description of the detector components and the event selections, readers are referred to the Supplemental Material of Ref. [四] and Refs. [园, 团].
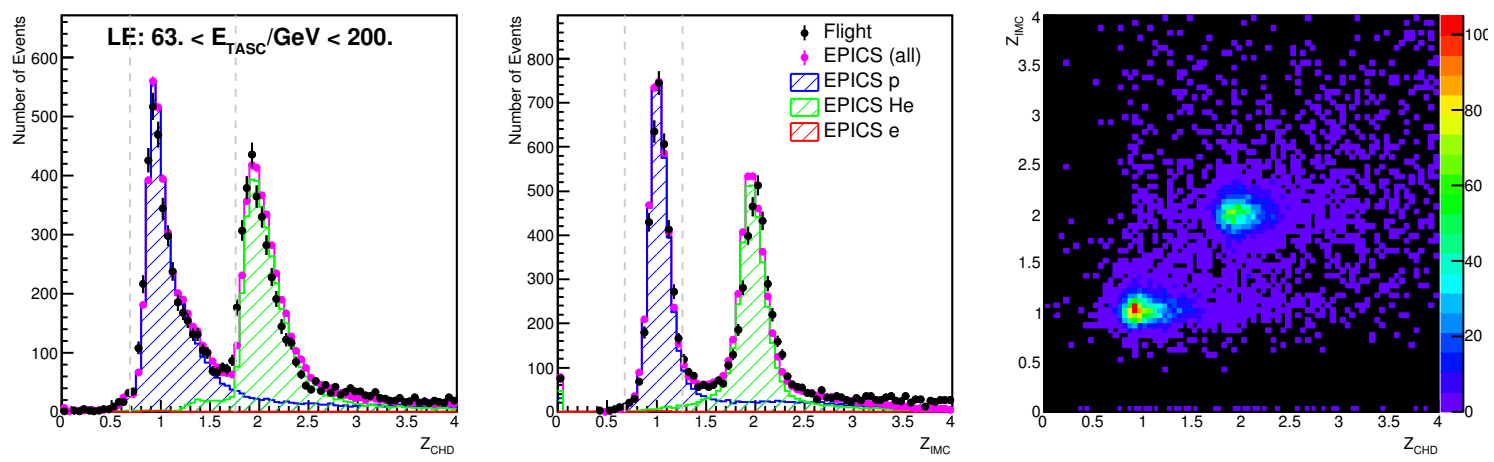

Figure 7: Examples of CHD/IMC charge distributions [29] corresponding to events with $63<E_{\mathrm{TASC}}<$ $200 \mathrm{GeV}$, where $E_{\mathrm{TASC}}$ denotes the TASC energy deposit sum. Left, center, and right panels show the CHD charge, IMC charge, and correlation between CHD and IMC charges, respectively. Gray dashed lines indicate the cut positions at $110 \mathrm{GeV}$.

Based on the preselected samples, charge identification was performed using CHD and IMC [BO]]. To mitigate backscattering effects, an energy-dependent charge correction to restore the nominal 
peak positions of protons and helium to $Z=1$ and 2, respectively, was applied separately to flight data, EPICS, FLUKA, and Geant4. Charge selection of proton and helium candidates was performed by applying simultaneous window cuts on CHD and IMC reconstructed charges. Typical examples of the resultant charge distributions are shown in Fig. $\square$. A clear separation of protons from helium can be observed.

Subsequently, a small contamination not exceeding 5\% was subtracted and energy unfolding was performed to correct for bin migration due to the for the limited energy resolution. Finally, taking into account the event selection efficiency, geometrical factor, and observation live time, the cosmic-ray proton flux was derived for each energy bin.

Figure $\mathbb{8}$ shows the proton spectrum measured with CALET in an energy range of $50 \mathrm{GeV}$ to $10 \mathrm{TeV}$ [R2], where current uncertainties that include statistical and systematic errors are bounded within the gray band. In Fig. 8, the CALET spectrum is compared with those from recent space experiments (PAMELA [B2, [33], AMS-02 [[23], and NUCLEON [28] ) and high-altitude balloon experiments (BESS-TeV [B]], ATIC-2 [24], CREAM-I [25], and CREAM-III [26]). Our spectrum is in good agreement with the very accurate magnetic spectrometer measurement by AMS-02 in the low-energy region, and the spectral behavior is consistent with measurements from calorimetric instruments in the higher-energy region.

The left panel of Fig. $Q$ shows the fits of the CALET proton spectrum with a single power law [ㅁ] ]. To study the spectral behavior, only the energy-dependent systematics are included in the data points. Red, blue, and magenta lines indicate the fitting results for energy intervals between 50 and $500 \mathrm{GeV}, 1$ and $10 \mathrm{TeV}$, and $50 \mathrm{GeV}$ and $10 \mathrm{TeV}$, respectively. The fit yields $\gamma_{1}=-2.81 \pm 0.03$

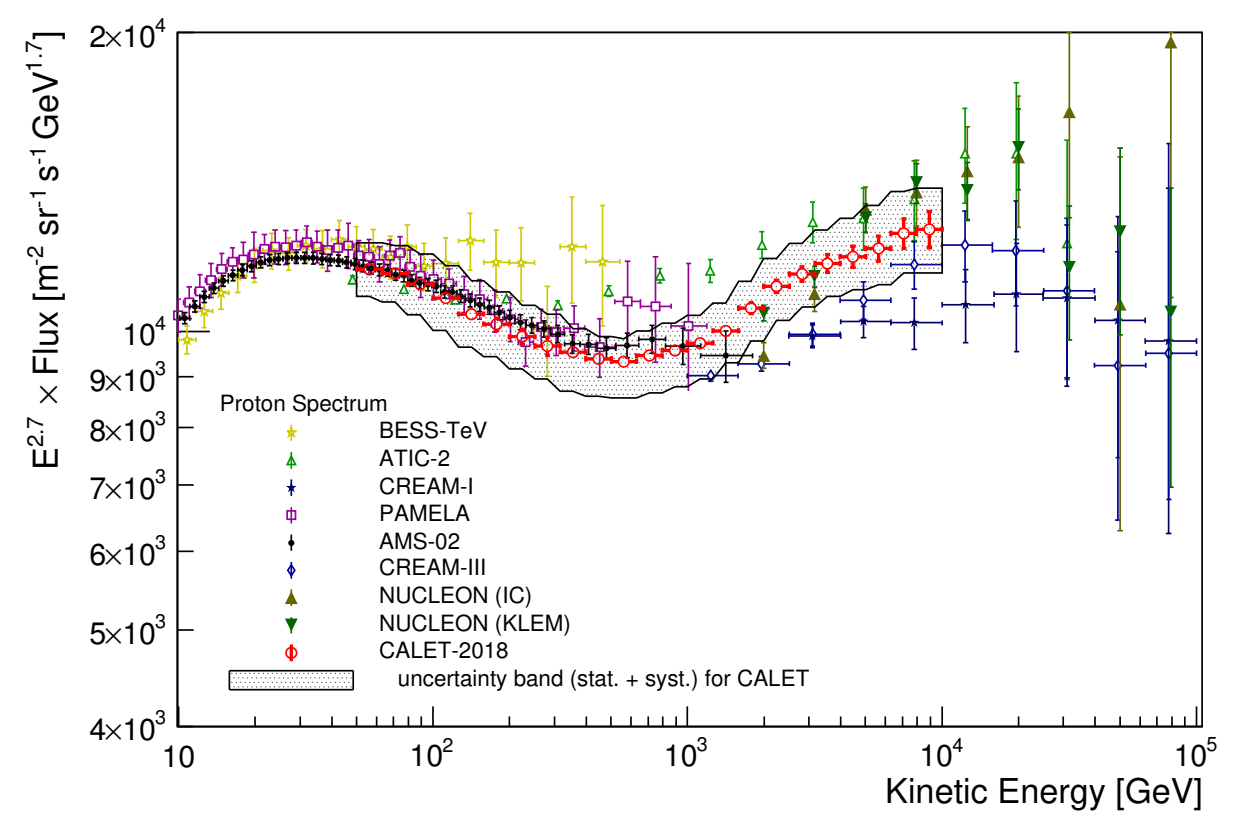

Figure 8: Cosmic-ray proton spectrum measured by CALET (red points) from $50 \mathrm{GeV}$ to $10 \mathrm{TeV}$ [RQ]. The gray band indicates the quadratic sum of statistical and systematic errors. Also plotted are recent direct measurements [24, 251, 231, 26, B], B2], [3], 28]]. 

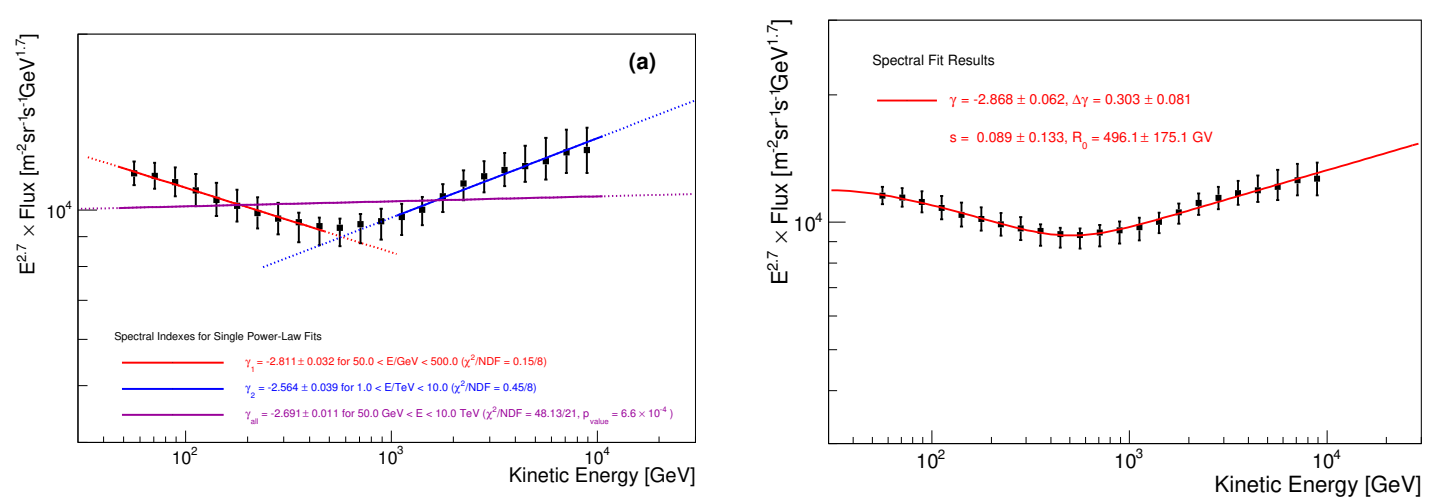

Figure 9: (Left) Fit of the CALET proton spectrum with single power-law functions [RQ]. Red, blue, and magenta lines indicate the fitting results for the energy ranges of 50 to $500 \mathrm{GeV}, 1$ to $10 \mathrm{TeV}$, and $50 \mathrm{GeV}$ to $10 \mathrm{TeV}$, respectively. (Right) Fit of the CALET proton spectrum with a smoothly broken power-law function [29] as defined in Eq. (3) in Ref. [34]. Statistical errors are shown in quadrature with systematic errors including only energy-dependent ones. $\mathrm{i}$

at lower energy (neglecting solar modulation effects) and $\gamma_{2}=-2.56 \pm 0.04$ at higher energy with good chi-square values. The fit over the whole range gives a large chi-square per degree of freedom, disfavoring the single power-law hypothesis by more than $3 \sigma$. Our spectrum can also be fitted with a smoothly broken power-law function [ [23, [35], as shown in the right panel of Fig. Q [ [29], resulting in a power-law index of $-2.87 \pm 0.06$ (including solar modulation effects) below the breakpoint rigidity, which is in good agreement with AMS-02 [D23]. A larger variation of the power-law index of $0.30 \pm 0.08$ and a higher breakpoint rigidity of $496 \pm 175 \mathrm{GV}$ than those for AMS-02 [23] are observed, though the latter is affected by relatively large error. These results may be important for the interpretation of the proton spectrum (e.g., [B6, [37, [38]) since they indicate a progressive hardening up to the $\mathrm{TeV}$ region, while being in good agreement with magnetic spectrometers in the $100 \mathrm{GeV}$ to sub-TeV region.

We measured, for the first time with an experimental apparatus in space, the cosmic-ray proton spectrum from $50 \mathrm{GeV}$ to $10 \mathrm{TeV}$, covering with a single instrument the whole energy range previously investigated by magnetic spectrometers (BESS-TEV, PAMELA, and AMS-02) and calorimetric instruments (ATIC, CREAM, and NUCLEON), which normally cover separate subranges of the region explored so far by CALET. Our observations confirm the presence of spectral hardening above a few hundred $\mathrm{GeV}$. Our spectrum is inconsistent with a single power law covering the whole range, while both the $50-500 \mathrm{GeV}$ and $1-10 \mathrm{TeV}$ subranges can be separately fitted with single power-law functions, with the spectral index of the lower (higher) energy region being consistent with AMS-02 [23] (CREAM-III [[26]) within errors. With the observation of a smoothly broken power law and an energy dependence of the spectral index, CALET's proton spectrum will shed light on the origin of the spectral hardening. 


\subsubsection{Nuclei spectra}

In the analysis of heavier nuclei, charge identification using CHD is critical, while it is also important to require consistency with IMC charge, which starts to saturate at above $Z \geq 10$. Figure 10 illustrates the charge identification capability of CALET based on the CHD data only, showing clear separation of nuclei up to iron and nickel.

Figure $\mathbb{W}$ shows the preliminary energy spectra of carbon, oxygen, neon, magnesium, silicon, and iron as a function of kinetic energy per particle with 962 days of operation up to May 31, 2018 [39]. These spectra are comparable with previous observations [40, 4], 42, 43], 444, 45, 46, 47, 48]. We note that the event selection that we applied here is based on a prelimi-

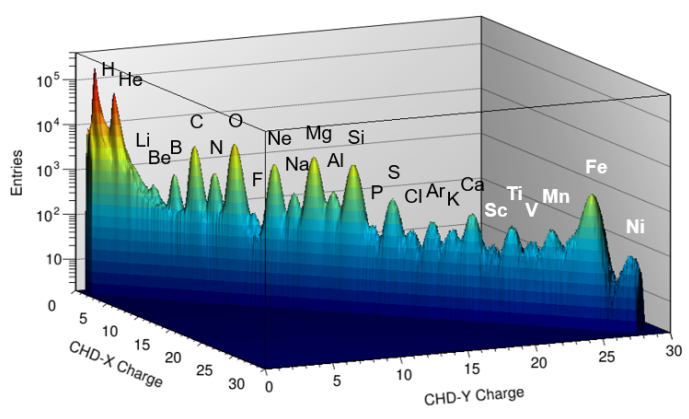

Figure 10: CHD charge separation [B9]. nary analysis, not what can eventually be achieved with CALET. Further studies are now underway to provide a more detailed analysis of these spectra [49]. The preliminary spectra illustrate the excellent capability of CALET to measure heavy ions with high statistics in a wide energy range. Further studies on an increased data set and a detailed systematic study will increase the sensitivity to detailed spectral features, which may be key for answering questions about galactic cosmic-ray acceleration and propagation.

\subsubsection{Ultra-heavy nuclei}

In addition to the nuclei spectra measurements, CALET has measured ultra-heavy cosmic-ray (UHCR) nuclei heavier than ${ }^{26} \mathrm{Fe}$. It has confirmed a dynamic range for measuring cosmic-ray nuclei of ${ }^{1} \mathrm{H}$ to ${ }^{40} \mathrm{Zr}$. A high-duty-cycle $(\sim 90 \%)$ UHCR trigger provides an expanded geometric acceptance that is $\sim 6 \times$ that for events fully contained by the calorimeter. In $\sim 5$ years, the UHCR trigger will collect a data set comparable to that so far collected by the balloon-borne SuperTIGER instrument. Preliminary CALET results presented at the last International Cosmic Ray Conference [50] were in reasonable agreement with SuperTIGER relative abundances of evencharge UHCR nuclei in a similar energy range. Both of these measurements are complemented by the $\sim 1 / 3$ smaller lower-energy space-based ACE-CRIS measurements. At this conference, we will present the current status and future plans for the CALET UHCR analysis [5]].

\subsection{Gamma Rays}

With a fully active calorimeter of 30-radiation-length thickness, CALET is capable of measuring gamma rays up to the TeV region. In addition to the HE trigger, CALET uses an LE- $\gamma$ trigger for gamma rays with primary energies down to $1 \mathrm{GeV}$. To avoid a large dead-time fraction, however, the LE- $\gamma$ trigger is activated only at low geomagnetic latitudes or following a gamma-ray burst (GRB) triggered onboard by the CALET gamma-ray burst monitor (CGBM).

\subsubsection{Instrument performance}




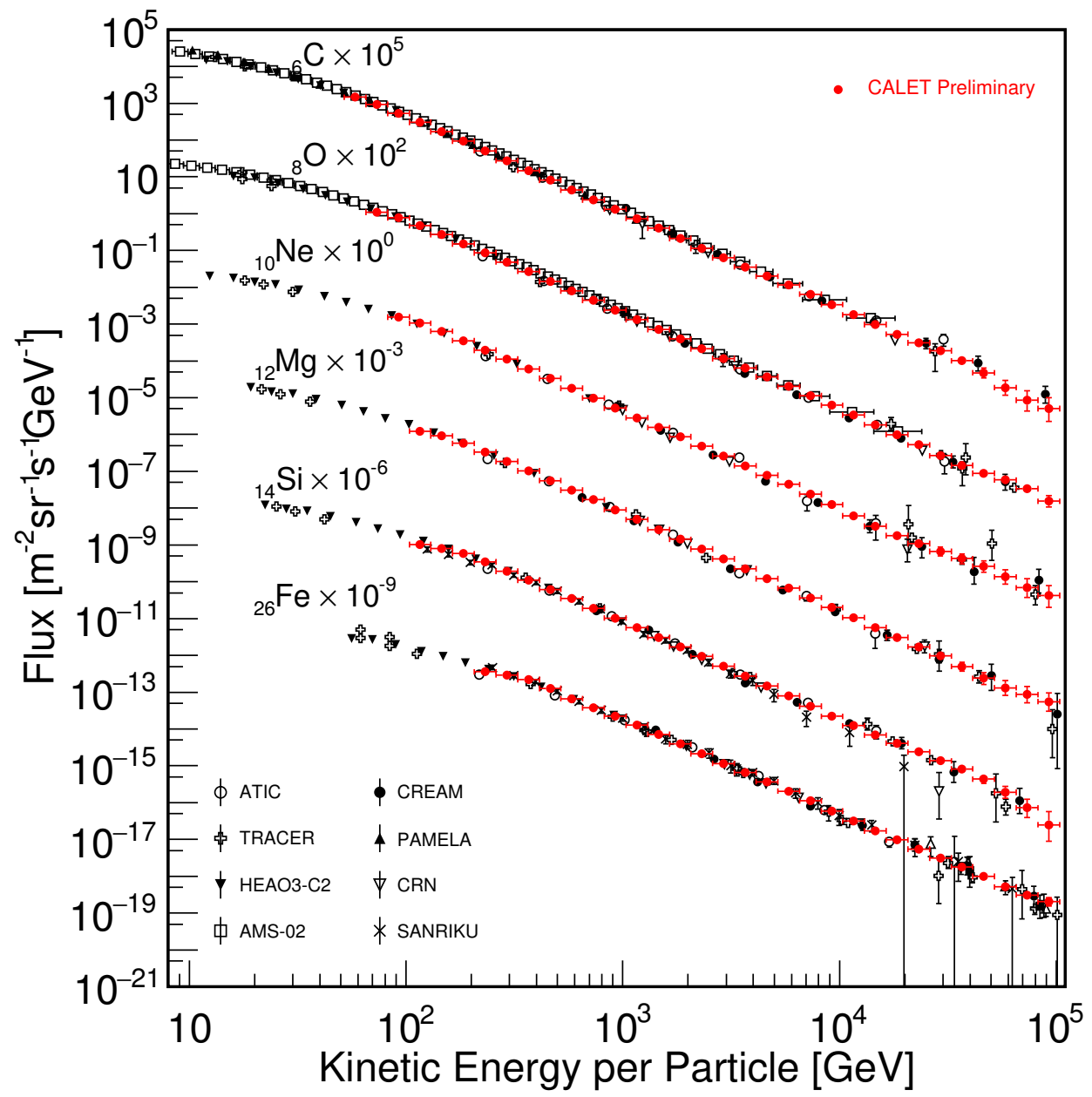

Figure 11: Preliminary energy spectra of carbon, oxygen, neon, magnesium, silicon, and iron [Bप] as functions of kinetic energy per particle after 962 days of CALET operation compared with previous observations [40, 41, 42, 43, 44, 45, 46, 47, 48]. Only statistical errors are shown.

The first 24 months of on-orbit scientific data provide valuable characterization of the performance of the calorimeter based on the analyses of the gamma-ray data set [52]. These analyses include optimization of event selection criteria, calculation of effective area, determination of the point spread function, confirmation of absolute pointing accuracy, observation of bright point sources, and study of diffuse components. Based on the developed analysis method, the

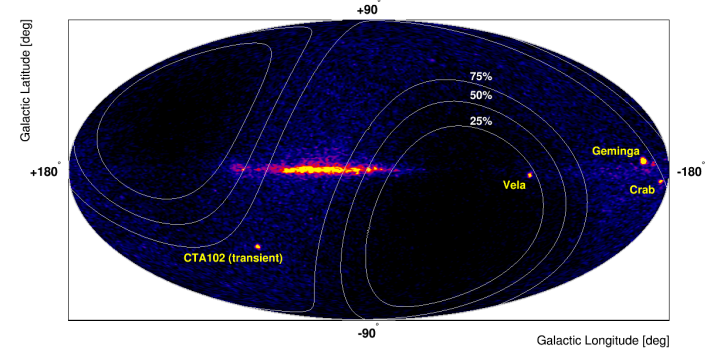

Figure 12: Gamma-ray sky map shown in a Mollweide projection of galactic coordinates. White contours show the relative level of exposure compared to the maximum on the sky.

CALET gamma-ray sky seen by the LE- $\gamma$ trigger is shown in Fig. [2], where galactic emission and bright gamma-ray sources are clearly identified. Point spread distributions and spectra of bright sources show very good agreement with simulation and Fermi-LAT's results, respectively [52], 
confirming our sensitivity to observe $\mathrm{GeV}$ gamma-rays.

\subsubsection{Observation of transients}

Gamma-ray transients are an important observational target for CALET. CGBM detected nearly 60 GRBs ( $\sim 20 \%$ short GRBs among them) per year in the energy range of $7 \mathrm{keV}$ to $20 \mathrm{MeV}$, as expected [ [3], [4]]. To search for GeV-energy counterpart emission from such sources also detected by other instruments, we checked the main-calorimeter data for gamma-ray candidates at the reported trigger time. For events based on CGBM, Swift, and Fermi/GBM triggers, no significant counterparts have been detected at this stage for time scales ranging from $1 \mathrm{~s}$ to $1 \mathrm{hr}$ [52]. The updated search of $\mathrm{GeV}$ gamma rays from confirmed GRBs will be presented at this conference [55]. Regarding the counterpart search for gravitational wave events, combined analyses of CGBM and the calorimeter were performed for GW151226, resulting in upper limits set on X-ray and gamma-ray counterparts [56]. Furthermore, complete search results of the CALET calorimeter on LIGO/Virgo's Observation Run 2 have been published recently [57]. We are performing counterpart searches for the ongoing LIGO/Virgo's Observation Run 3. The results will be presented at this conference [58].

\section{Summary and Prospects}

CALET was successfully launched on August 19, 2015, and detector performance for scientific observation has been very stable since October 13, 2015. Careful calibrations using noninteracting protons and helium events have been successfully carried out, and the linearity of the energy measurements up to $10^{6}$ MIPs was established based on observed events [ [ $\mathbb{Q}, \mathbb{Q}$ ].

The all-electron spectrum [ [ $]$ has been published with an extended energy range of $11 \mathrm{GeV}$ to $4.8 \mathrm{TeV}[\mathrm{U}]$ and improved statistics. Figure $\mathbb{\square 3}$ presents the current situation of all-electron spectrum direct measurements in space. Five or more years of observations with CALET will triple the statistics [5], which together with a reduction of systematic errors based on a better understanding of the detector with an increased amount of flight data will lead to significantly improved precision. This will allow a refined study of the possible fine structures around a few hundred $\mathrm{GeV}$ and $\sim 1 \mathrm{TeV}$, which are currently not significant, and

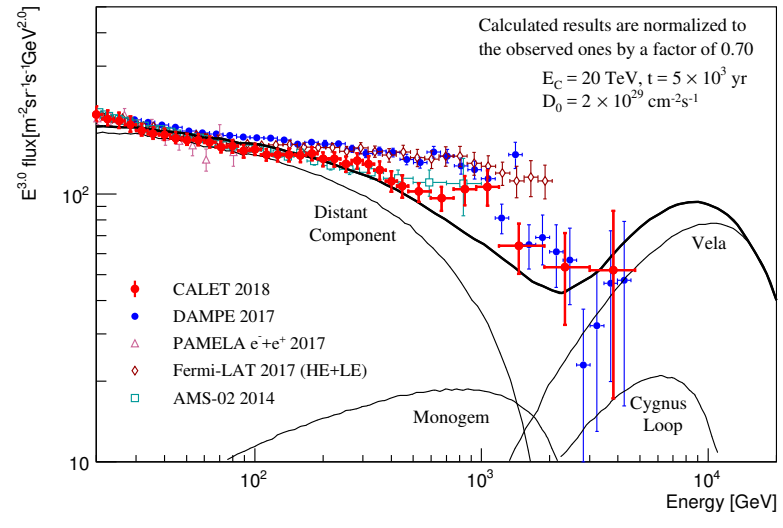

Figure 13: Current situation of all-electron spectrum directly measured in space, together with the model calculation [ए]], where the main model parameters are shown in the plot. this might shed light on the origin of the positron excess. An extension of the high-energy reach using improved statistics and electron separation methods might for the first time reveal a charged cosmic-ray signature of a local accelerator. 
Extension of the lower-energy reach is also in progress to study solar modulation and shorter time flux variations such as Forbush decreases [60].

In our first publication of the proton spectrum [29], we identified progressive hardening up to the $\mathrm{TeV}$ region, which is in good agreement with magnetic spectrometers in the $100 \mathrm{GeV}$ to sub$\mathrm{TeV}$ region [G]. In the near future, improved statistics and better understanding of the instrument based on the analysis of additional flight data over the 5 or more years of observations might reveal a charge-dependent energy cutoff possibly due to the acceleration limit in supernova remnants in proton and helium spectra, or set important constraints on the acceleration models.

As to the heavier nuclei, preliminary results on primary and secondary nuclei up to $Z=26$ and their ratios (for example, boron to carbon) [62, [39, 63, 69] demonstrate CALET's wide dynamic range of energy measurement from $1 \mathrm{GeV}$ to $1 \mathrm{PeV}$ and the accuracy of its charge determination capability. These results, which will be published in the near future, will address important questions in cosmic-ray physics, such as the universality of the widely observed spectral hardening and the energy dependence of the diffusion coefficient. The relative abundance of the ultra-heavy nuclei up to $Z=40$ was also analyzed [ 5$]$ ]

There is also significant progress in CALET's gamma-ray analysis. Based on the data acquired in 2 years on orbit, the performance of the gamma-ray measurements has been characterized [52]. These results confirm the capability of CALET to observe gamma rays in the energy range of $\sim 1 \mathrm{GeV}$ to over $100 \mathrm{GeV}$. CALET's current results of the electromagnetic counterpart search for gravitational wave events [56, 57] show the great potential of follow-up observations during LIGO/Virgo's upcoming Observation Run 3. The continuous GeV gamma-ray sky observation with CALET complements the coverage by other missions and may help to identify unexplored high-energy emissions from future transient events. Watching for various transient phenomena, including those in gamma rays, is an important task for CALET as an on-orbit observation strategy $\left[{ }_{55}, 58\right]$. Through the detection of many events of $\mathrm{MeV}$ electrons originating from the radiation belt [64], a phenomenon called relativistic electron precipitation, space weather was added as an additional observational target for CALET after the start of on-orbit operations [65]].

The excellent performance of CALET and the outstanding quality of the data suggest that 5 or more years of observations will most likely provide a wealth of interesting new results.

\section{Acknowledgment}

We gratefully acknowledge JAXA's contributions to the development of CALET and to the operations onboard the International Space Station. We also wish to express our sincere gratitude to ASI and NASA for their support of the CALET project. This work was supported in part by JSPS KAKENHI Grant Numbers 26220708, 16K05382, 17H02901, 19H04617, 19H05112, 19H05608, and by the MEXT-Supported Program for the Strategic Research Foundation at Private Universities (2011-2015) (No. S1101021) at Waseda University. The CALET effort in the United States is supported by NASA through grants nos. NNX16AB99G, NNX16AC02G, and NNH14ZDA001NAPRA-0075.

\section{References}

[1] K. Yamaoka et al., in Proc. 7th Huntsville Gamma-Ray Burst Symposium, GRB 2013 (2013), p. 41 
[2] S. Torii et al. (CALET Collaboration), in Proceeding of Science (ICRC2015) 581 (2015)

[3] S. Torii et al. (CALET Collaboration), in Proceeding of Science (ICRC2017) 1092 (2017)

[4] P.S. Marrocchesi et al., Nucl. Instrum. Methods Phys Res., Sect. A 659, 477 (2011)

[5] P.S. Marrocchesi et al. (CALET Collaboration), in Proc. of 33rd ICRC (2013), p. 362

[6] O. Adriani et al. (CALET Collaboration), Phys. Rev. Lett. 119, 181101 (2017)

[7] O. Adriani et al. (CALET Collaboration), Phys. Rev. Lett. 120, 261102 (2018)

[8] Y. Asaoka, Y. Ozawa, S. Torii et al. (CALET Collaboration), Astropart. Phys. 100, 29 (2018)

[9] Y. Asaoka, Y. Akaike, Y. Komiya, R. Miyata, S. Torii et al. (CALET Collaboration), Astropart. Phys. 91, 1 (2017)

[10] J. Nishimura et al., ApJ 238, 394 (1980)

[11] T. Kobayashi, Y. Komori, K. Yoshida, J. Nishimura, Astrophys. J. 601, 340 (2004)

[12] O. Adriani et al., Nature 458, 607 (2009)

[13] L. Accardo et al. (AMS Collaboration), Phys. Rev. Lett. 113, 121101 (2014)

[14] G. Ambrosi et al. (DAMPE Collaboration), Nature 552, 63 (2017)

[15] O. Adriani et al., La Rivista del Nuovo Cimento 40, 473 (2017)

[16] S. Abdollahi et al. (The Fermi-LAT Collaboration), Phys. Rev. D 95, 082007 (2017)

[17] M. Aguilar et al. (AMS Collaboration), Phys. Rev. Lett. 113, 221102 (2014)

[18] F. Aharonian et al. (H.E.S.S. Collaboration), Phys. Rev. Lett. 101, 261104 (2008)

[19] F. Aharonian et al. (H.E.S.S. Collaboration), Astron Astrophys. 508, 561 (2009)

[20] H. Motz et al. (CALET Collaboration), in Proceeding of Science (ICRC2019), in press (2019)

[21] A.R. Bell, K.M. Schure, B. Reville, G. Giacinti, Mon. Not. R. Astron Soc. 431, 415 (2013)

[22] O. Adriani et al., Science 332, 69 (2011)

[23] M. Aguilar et al. (AMS Collaboration), Phys. Rev. Lett. 114, 171103 (2015)

[24] A. Panov et al., Bull. Russ. Acad. Sci. Phys. 71, 494 (2007)

[25] Y. Yoon et al., Astrophys. J. 728, 122 (2011)

[26] Y. Yoon et al., Astrophys. J. 839, 5 (2017)

[27] E. Atkin et al., JCAP 07, 020 (2017)

[28] E. Atkin et al., JETP Letters 108, 5 (2018)

[29] O. Adriani et al. (CALET Collaboration), Phys. Rev. Lett. 122, 181102 (2019)

[30] P.S. Marrocchesi et al. (CALET Collaboration), in Proceeding of Science (ICRC2017) 156 (2017)

[31] S. Haino et al., Phys. Lett. B 594, 35 (2004)

[32] O. Adriani et al., Phys. Rept. 544, 323 (2014)

[33] O. Adriani et al., Riv. Nuovo Cim. 40, 1 (2017)

[34] M. Aguilar et al. (AMS Collaboration), Phys. Rev. Lett. 114, 171103 (2015) 
[35] L. Glesson, W. Axford, Astrophys. J. 154, 1011 (1968)

[36] P. Blasi, E. Amato, P.D. Serpico, Phys. Rev. Lett. 109, 061101 (2012)

[37] R. Aloisio, P. Blasi, J. Cosmol. Astropart. Phys. 07, 001 (2013)

[38] C. Evoli, P. Blasi, G. Morlino, R. Aloisio, Phys. Rev. Lett. 121, 021102 (2018)

[39] Y. Akaike et al. (CALET Collaboration), J. Phys. Conf. Series 1181, 012042 (2018)

[40] M. Aguilar et al. (AMS Collaboration), Phys. Rev. Lett. 119, 251101 (2017)

[41] M. Aguilar et al. (AMS Collaboration), Phys. Rev. Lett. 120, 021101 (2018)

[42] A. Panov et al., Bull. Russ. Acad. Sci. Phys. 73, 564 (2009)

[43] H. Ahn et al., Astrophys. J. 707, 593 (2009)

[44] A. Obermeier et al., ApJ 724, 14 (2011)

[45] A. Adriani et al., ApJ 791, 93 (2014)

[46] J. Engelmann et al., Astron. Astrophys. 233, 96 (1990)

[47] D. Muller et al., ApJ 374, 356 (1991)

[48] M. Ichimura et al., Phys. Rev. D 48, 1949 (1993)

[49] Y. Akaike et al. (CALET Collaboration), in Proceeding of Science (ICRC2019), in press (2019)

[50] R. Brian, Y. Akaike et al. (CALET Collaboration), in Proceeding of Science (ICRC2017) 180 (2017)

[51] B. Rauch, B. Binns et al. (CALET Collaboration), in Proceeding of Science (ICRC2019), in press (2019)

[52] N. Cannady, Y. Asaoka et al. (CALET Collaboration), ApJS 238, 5 (2018)

[53] K. Yamaoka et al. (CALET Collaboration), in Proceeding of Science (ICRC2017) 613 (2017)

[54] Y. Kawakubo et al. (CALET Collaboration), in Proceeding of Science (ICRC2019), in press (2019)

[55] N. Cannady et al. (CALET Collaboration), in Proceeding of Science (ICRC2019), in press (2019)

[56] O. Adriani et al. (CALET Collaboration), ApJL 829, L20 (2016)

[57] O. Adriani et al. (CALET Collaboration), ApJ 863, 160 (2018)

[58] M. Mori, Y. Asaoka et al. (CALET Collaboration), in Proceeding of Science (ICRC2019), in press (2019)

[59] S. Torii et al. (CALET Collaboration), in Proceeding of Science (ICRC2019), in press (2019)

[60] S. Miyake et al. (CALET Collaboration), in Proceeding of Science (ICRC2019), in press (2019)

[61] P. Marrocchesi et al. (CALET Collaboration), in Proceeding of Science (ICRC2019), in press (2019)

[62] Y. Akaike et al. (CALET Collaboration), in Proceedings of Science (ICRC2017) 181 (2017)

[63] P. Maestro et al. (CALET Collaboration), in Proceeding of Science (ICRC2019), in press (2019)

[64] R. Kataoka et al., Geophys. Res. Lett. 43, doi:10.1002/2016GL068930 (2016)

[65] A. Bruno, G. de Nolfo, A. Ficklin et al. (CALET Collaboration), in Proceeding of Science (ICRC2019), in press (2019) 